\title{
Development of a Compact X-ray Source and Super-sensitization of Photo Resists for Soft X-ray Imaging
}

\author{
Tomoko Gowa ${ }^{1}$, Naoyuki Fukutake ${ }^{1}$, Yoshimasa Hama ${ }^{1}$, Kentaro Hizume ${ }^{1}$, \\ Takashi Kashino $^{1}$, Shigeru Kashiwagi ${ }^{2}$, Ryunosuke Kuroda ${ }^{3}$, Akihiko Masuda ${ }^{1}$, \\ Akihiro Oshima ${ }^{2}$, Taku Saito', Kazuyuki Sakaue ${ }^{1}$, Kunio Shinohara ${ }^{1}$, \\ Tomohiro Takahashi ${ }^{1}$, Tatsuya Urakawa ${ }^{1}$, Kiminori Ushida ${ }^{4}$, Masakazu Washio ${ }^{1}$ \\ 1: Research Institute for Science and Engineering, Waseda University, \\ 3-4-1 Okubo, Shinjuku-ku, Tokyo 169-8555, Japan \\ 2: Institute of Scientific and Industrial Research, Osaka University, \\ 8-1 Mihogaoka, Ibaragi, Osaka 567-0047, Japan \\ 3: National Institute of Advanced Industrial Science and Technologies, \\ 1-1 Umezono, Tsukuba, Ibaraki 305-0045, Japan \\ 4: RIKEN, 2-1 Hirosawa, Wako, Saitama 351-0198, Japan
}

A compact soft X-ray source via inverse Compton scattering has been developed at Waseda University. The energies of the generated X-rays are within "water window" region $(250-500 \mathrm{eV})$ and development of a soft X-ray microscope is expected which can get the elemental mapping of carbon and/or nitrogen without dehydration. We have studied to develop a high resolution soft $\mathrm{X}$-ray imaging system with photo resists for nanoscale observation. However, the yield of generated X-rays had been too small for the practical use of the soft $\mathrm{X}$-ray microscopy. To enhance the X-ray yield, we have upgraded the generation system and succeeded in increasing the detected photons 10 -fold. Total generated photons were estimated to be over $1.5 \mathrm{E}+5$ photons/s. Also, super-sensitization of photo resists has been attempted to reduce the required X-ray amount. By irradiating quasi-monochromatic X-rays in the water window region from synchrotron radiation at BL12 of the SAGA-LS, the sensitivity of a deep-UV photo resist, TDUR-P722 (Tokyo Ohka Kogyo Co., Ltd) was evaluated. After UV light (including $254 \mathrm{~nm}$ ) exposure up to the sensitivity threshold and baking as PEB process, it was found that the resist becomes more sensitive. This UV "pre-exposure" method reduced the required $400 \mathrm{eV}$ X-ray amount by over $65 \%$. Keyword: photo resist, inverse Compton scattering, soft X-ray microscope

\section{Introduction}

Short-pulse soft X-ray sources are now widely required in various fields, such as medical, biological, chemical and material science. A compact soft X-ray source via inverse Compton scattering using a photo-cathode RF-gun and a picosecond laser system has been developed at Waseda University [1, 2]. Inverse Compton scattering is the inverse energy transfer process of general Compton scattering between

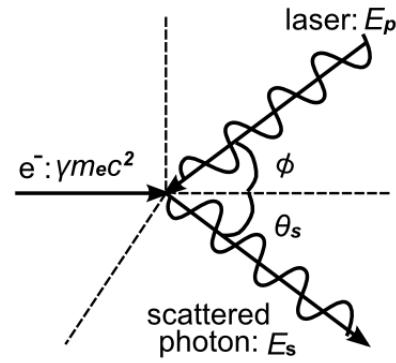

Lab. Frame

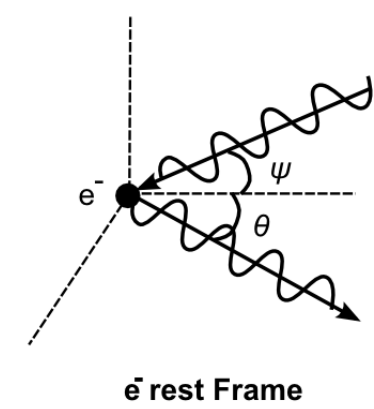

Figure 1. Inverse Compton scattering in the laboratory and electron rest frames 
relativistic electrons and low energy photons such as a laser light. The laser light, which collides with high energy electrons, is scattered as X-rays by gaining its energy [3].

Figure 1 shows the coordinates of the inverse Compton scattering in the laboratory and electron rest frames. When the electron rest mass $\left(m_{e}\right)$, the electron energy $\left(E_{b}\right)$, Lorentz factor $\gamma\left(E_{b}=\gamma m_{e} c^{2}\right)$, and the incident laser photon energy $\left(E_{p}\right)$ are defined in the laboratory frame, the energy of scattered photons $\left(E_{s}\right)$ is given as

$$
E_{s}=\frac{m_{e} c^{2} \gamma^{2}(1+\beta \cos \phi)(1+\beta \cos \theta) E_{p}}{m_{e} c^{2}+\gamma(1+\cos \theta)(1+\beta \cos \phi) E_{p}}
$$

where $\theta$ is the scattered angle in the electron rest frame and $\phi$ is the crossing angle in the laboratory frame.

Using a Nd:YLF (1047 $\mathrm{nm})$ laser beam and a $5 \mathrm{MeV}$ class electron beam generated from a photo-cathode RF-gun system, we have already succeeded in generating inverse Compton X-rays [2]. The energies of the generated X-rays are within so-called "water window" region $(250-500 \mathrm{eV})$ where the $\mathrm{X}$-ray absorption coefficient of water is much less than those of carbon and nitrogen as shown in Figure 2 [4]. Hence, development of a soft X-ray microscope is expected which can get the elemental mapping of a living cell or hydro-gels without dehydration [5]. As described in Equation (1), the X-ray energy is tunable by changing the electron beam energy and/or the collision angle. In addition, quasi-monochromatic X-ray beam can be

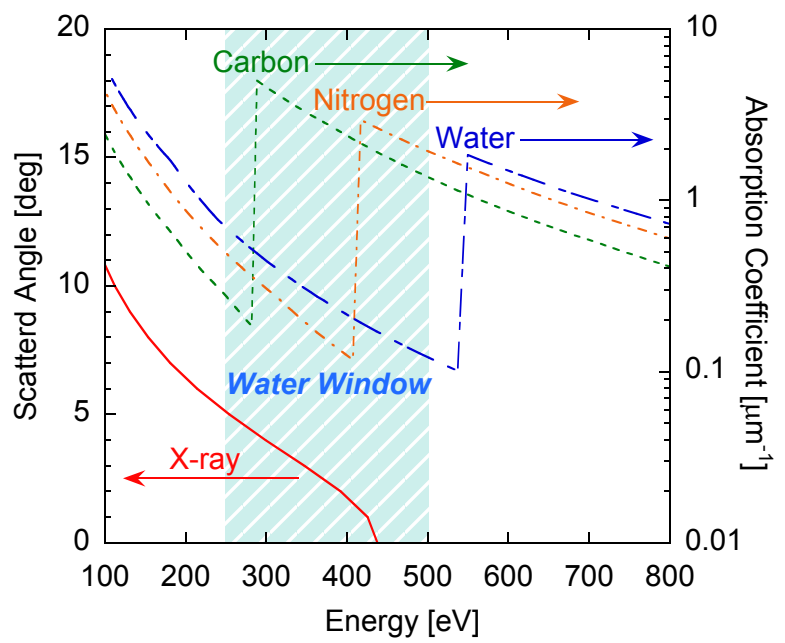

Figure 2. The water window region [4] and calculated energy distribution of generated X-rays with $5 \mathrm{MeV}$ electron beam and $1047 \mathrm{~nm}$ laser collision ( $\phi=20 \mathrm{deg}$ ). obtained by selecting the scattered angle. Its short pulse length and compactness of the instruments are also important advantages of inverse Compton X-rays [2].

For the soft X-ray microscopy, there're several imaging types: contact, projection, scanning and so on [5]. We have studied to develop a contact microscope with photo resists as the detector, which is the conceptually-simplest and expected to have high resolution [6]. By placing the resist behind the object and irradiating X-rays, the object's profile and X-ray absorption information can be transferred to the resist as the pattern and its depth. The resolution of the resist, which is as high as several $\mathrm{nm}$, is critical for the spacial resolution of the contact microscopy [7]. Therefore, by examining the resist surface with suitable profilometers such as an atomic force microscope (AFM), nanoscale imaging and the elemental mapping will be possible.

For the practical application to the X-ray microscopy, it is estimated that about $1 \mathrm{E}+11$ photons $/ \mathrm{mm}^{2}$ are required to get an absorption contrast image of hydrated specimens with resolution of $100 \mathrm{~nm} \mathrm{[8,9].} \mathrm{However,} \mathrm{at}$ Waseda University, the yield of generated $\mathrm{X}$-rays had been too small. Therefore, we have tried to solve this problem through two approaches: the enhancement of the generated $\mathrm{X}$-ray yield by upgrading the generation system and the reduction of the required $\mathrm{X}$-rays for the imaging by super-sensitization of the resists.

\section{Experimental}

\subsection{X-ray Generation Setup}

Figure 3 shows a schematic diagram of the experimental setup of inverse Compton X-ray generation in the present study $[10,11]$. The system is constructed on a $2.5 \times 2 \mathrm{~m}^{2}$ optical table, including all the beam lines and a picosecond Nd:YLF laser system (Pulrise-V: Sumitomo Heavy Industry Co.). The RF-gun is composed of BNL-type 1.6 cells S-band RF cavities and copper cathode. The Nd:YLF fundamental IR laser $(1047 \mathrm{~nm})$ is used as the incident laser for the scattering, and its $4^{\text {th }}$ harmonic UV laser $(262 \mathrm{~nm})$ is for generating electrons by irradiating the photo-cathode. The IR laser passes through a flash lamp pumped Nd:YLF amplifier system and an 


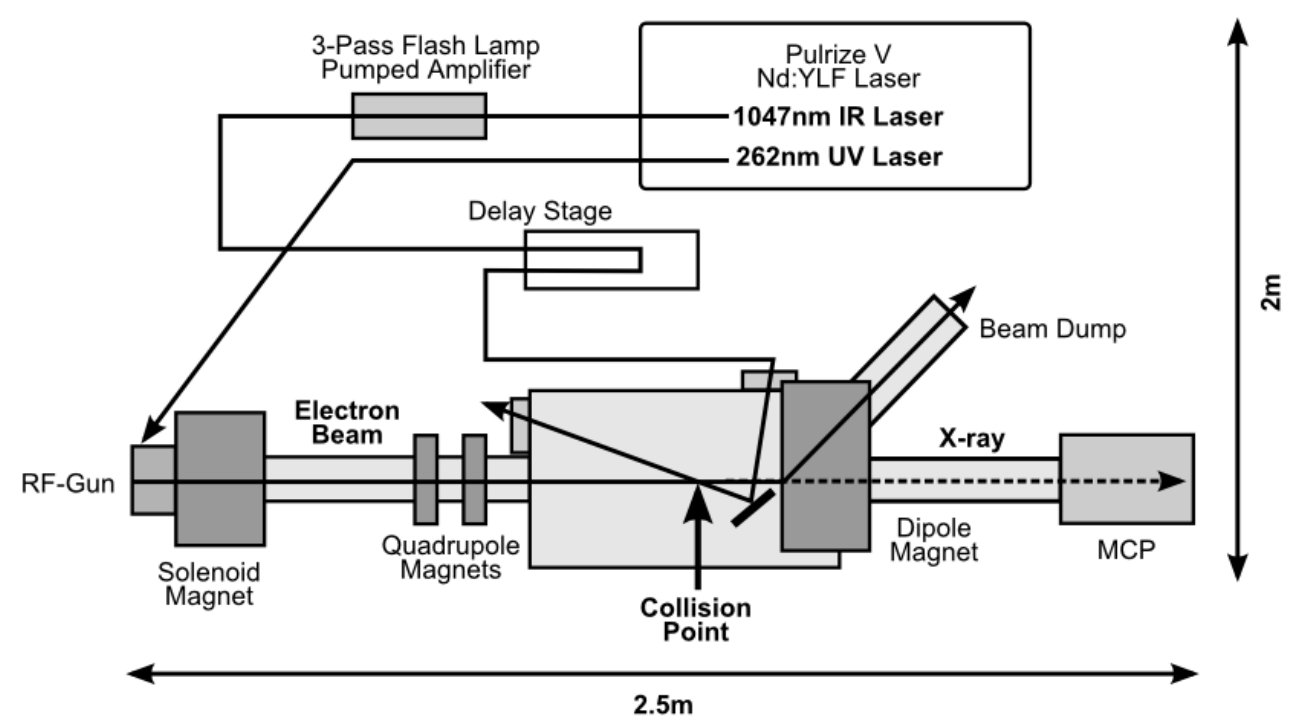

Figure 3. Schematic diagram of the experimental setup for the X-ray generation at Waseda University

optical delay stage which controls the timing of the electron-laser interaction. Since both IR and UV lasers are generated from the same laser system, the interaction timing is able to synchronize easily. We can choose the collision angle, i.e. X-ray energy, by tuning an IR mirror located before the collision point. For the X-ray detection, a micro channel plate, MCP (F4655-10: Hamamatsu Photonics K.K.), is installed after the electron beam is separated from the generated X-rays.

\subsection{Resist Sample Preparation}

For the soft X-ray imaging, both high sensitivity and high resolution are required for the photo resists. As one of the potential candidates, a positive deep-UV photo resist, TDUR-P722 (Tokyo Ohka Kogyo Co., Ltd) was selected. TDUR-P722 is a chemically amplified photo resist which is composed of a basic polymer resin and a photo-acid generator. In this study, the resist was spin-coated on the silicon wafers and pre-baked at $140{ }^{\circ} \mathrm{C}$ for $90 \mathrm{~s}$ on a hot plate. The thickness of the resist was approximately $600 \mathrm{~nm}$ when the spin-coating speed and duration time were $2000 \mathrm{rpm}$ and $60 \mathrm{~s}$, respectively. After the exposure, the post exposure bake (PEB) was performed at $140{ }^{\circ} \mathrm{C}$ for $90 \mathrm{~s}$, and the samples were developed by alkaline aqueous based developer, NMD-3 (Tokyo Ohka Kogyo Co., Ltd), at $23{ }^{\circ} \mathrm{C}$ for $65 \mathrm{~s}$. After that, they were rinsed by the de-ionized water for $30 \mathrm{~s}$ and post-baked at $100{ }^{\circ} \mathrm{C}$ for $60 \mathrm{~s}$.

\subsection{Exposure Condition}

The photo sensitivity was obtained by the resist pattern depth dissolved in the developer measured with an AFM (SPI3000: SII). First, the sensitivity to the deep-UV light was measured, because the resist is originally made for the deep-UV lithography. As a light source, a low-pressure mercury vapor lamp (including $254 \mathrm{~nm}$ ) was used, and all the processes from the spin-coating to the development were performed under ambient conditions. A $40 \mu \mathrm{m}$ square pattern $\mathrm{Cu}$ mesh mask was set on the resist samples to transfer the pattern. The exposure amount was measured with an UV power meter (C9536, H9535-254: Hamamatsu Photonics K.K.).

Secondly, to evaluate the sensitivity to the water window region, the irradiation experiment was carried out at BL12 of the SAGA Light Source (SAGA-LS). The SAGA-LS is a $1.4 \mathrm{GeV}$ storage ring which average stored current is $200 \mathrm{~mA}$ [12]. At BL12, quasi-monochromatic X-rays from 40 $\mathrm{eV}$ to $1500 \mathrm{eV}$ are obtained through a varied line spacing plane grating monochromator. The flux at the sample position is over $1 \mathrm{E}+9$ photons/s in the water window region. In this experiment, the total fluence was obtained by integrating the flux which is estimated by the photocurrent from Au mesh. Then, the actual fluence on the samples was recalculated considering the spacial distribution of the $\mathrm{X}$-ray beam. In the irradiation process, a 70 $\mu \mathrm{m}$ square pattern Ni mesh was set as a mask. The base pressure in the irradiation chamber 
was below 10E-6 Pa, and the sample preparation and the development were performed under ambient conditions.

\section{Results and Discussion}

\subsection{Soft X-ray Generation}

At Waseda University, we achieved a first observation of the inverse Compton X-rays in 2004 [2]. Since that time, we have been upgrading our system to improve the X-ray quality with an incident laser amplification system, background reduction, an electron beam optimization and so on $[10,11]$. The recent parameters of both electron and laser beams at the collision point are summarized in Table 1. With these modifications, the $\mathrm{X}$-ray photons detected by the MCP have increased 10 -fold to reach $1.6 \mathrm{E}+3$ photons/s. The maximum energy of generated X-rays was calculated to be about $370 \mathrm{eV}$. Good signal to noise ratio $6.2 \mathrm{ps}$ (RMS) short pulse $\mathrm{X}$-rays was obtained, and the number of total generated X-rays was estimated to be over $1.5 \mathrm{E}+5$ photons/s. Moreover, we succeeded in generating soft X-rays stably over 12 hours [11].

Table 1. Recent experimental parameters of the electron beam and the laser beam at the collision point

\begin{tabular}{llll}
\hline \hline \multicolumn{2}{c}{ Electron beam } & \multicolumn{2}{c}{ Laser beam } \\
\hline Energy & $4.6 \mathrm{MeV}$ & Wavelength & $1047 \mathrm{~nm}$ \\
Bunch Charge & $350 \mathrm{pC}$ & Pulse energy & $36 \mathrm{~mJ}$ \\
Size (horizontal) & $251 \mu \mathrm{m}$ & Size (horizontal) & $42 \mu \mathrm{m}$ \\
Size (vertical) & $56 \mu \mathrm{m}$ & Size (vertical) & $42 \mu \mathrm{m}$ \\
Bunch length & $10 \mathrm{ps}(\mathrm{FWHM})$ & Pulse duration & $10 \mathrm{ps}$ (FWHM) \\
\hline \hline
\end{tabular}

Still, the generated X-rays are not enough for the soft X-ray microscopy. Therefore, we are planning to develop a multi-pulse inverse Compton scattering system [13]. With multi-pulsed electron and laser beams, the generated X-rays would increase about 7000 -fold in simulations. For the multi-bunch electron beam generation, we have already installed a new RF-gun with $\mathrm{Cs}_{2} \mathrm{Te}$ cathode which has 100 -fold higher quantum efficiency than the copper cathode.

\subsection{Sensitivity Measurement}

Figure 4 shows a sensitivity curve of the resist indicating the normalized depth of the pattern formed on the resist in relation to the deep-UV light exposure. The error bars indicate the standard deviation of the measured value for five samples.

The resist has the threshold of exposure for dissolving in the developer, i.e., obtaining the image. As shown in Figure 4, the sensitivity threshold to deep-UV light was approximately $2.6 \mathrm{~mJ} / \mathrm{cm}^{2}$. This result shows relatively high sensitivity because the mask pattern is as large as $40 \mu \mathrm{m}$ square and it can be considered as a flat-exposure. The oxidation would also be a factor since the experiment was carried out under ambient conditions.

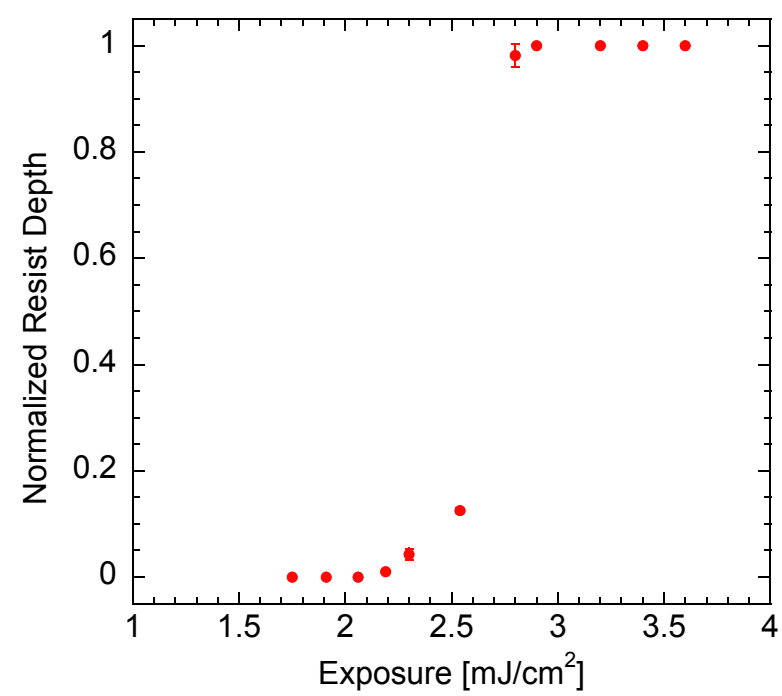

Figure 4. Sensitivity curve of TDUR-P722 to $254 \mathrm{~nm}$ deep-UV light exposure

In the same way, the sensitivity to the water window region was evaluated. Figure 5 shows the transferred mask pattern on the resist after irradiating $250 \mathrm{eV}$ and $400 \mathrm{eV}$ $\mathrm{X}$-rays. Also, similar result was obtained for $500 \mathrm{eV} \mathrm{X}$-ray irradiation, which means the resist is sensitive to throughout the water window region. In this study, the sensitivity at $400 \mathrm{eV}$ was especially measured because the X-rays which can be stably generated with our present system are around $400 \mathrm{eV}$.
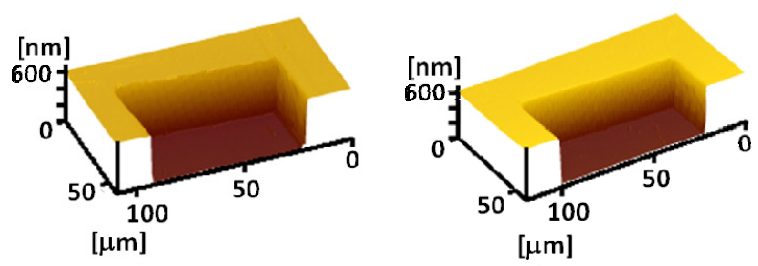

Figure 5. The resist surface observed with AFM after irradiating $250 \mathrm{eV}$ (left) and $400 \mathrm{eV} \mathrm{X}$-rays (right)

At $400 \mathrm{eV}$ (3.1 $\mathrm{nm}$ in wavelength), the sensitivity threshold was approximately 
$3.3 \mathrm{E}+11$ photons $/ \mathrm{mm}^{2}$, i.e. $2.1 \mathrm{~mJ} / \mathrm{cm}^{2}$. On the other hand, to $250 \mathrm{eV}(4.96 \mathrm{~nm})$, the threshold was over $3.0 \mathrm{E}+12$ photons $/ \mathrm{mm}^{2}$, i.e. $13 \mathrm{~mJ} / \mathrm{cm}^{2}$. The sensitivity to the $400 \mathrm{eV}$ $\mathrm{X}$-rays is about 10 -fold higher than to the 250 $\mathrm{eV}$. This can be explained by the X-ray absorption coefficient of carbon which is the main constituent element of the resist. This result indicates that the required irradiation amount varies widely in the water window region which contains the $\mathrm{K}$-shell absorption edge of carbon. The detail of this difference in sensitivity and its reaction mechanisms will be reported in the near future.

\subsection{UV Pre-Exposure Method}

Higher sensitivity is necessary for the resist materials to reduce the required X-rays because the yield of generated X-rays is still inadequate. Even if the generated X-rays will be enough for the imaging, higher sensitivity is important because short exposure time will avoid image blurring. Also, considering the radiation damage to the objects, the required fluence should be reduced. Therefore, we attempted the super-sensitization of the TDUR-P722.

After the UV light exposure up to the threshold and baking as PEB process, we found that the resist becomes more sensitive to the deep-UV light. The exposure up to the threshold, "pre-exposure", generates certain amount of acid. During the baking process which is performed just like PEB, the generated acid would cause chemical reaction although it wouldn't be enough for the resist to dissolve in the developer. This chemical reaction caused by the pre-exposure would raise the sensitivity of the resist.

Figure 6 shows the sensitivity curve of the $2.0 \mathrm{~mJ} / \mathrm{cm}^{2} \mathrm{UV}$ pre-exposed resist samples, in comparison with that of without pre-exposure (blank). The photo sensitivity to the deep-UV light was successfully improved. The threshold became approximately $0.4 \mathrm{~mJ} / \mathrm{cm}^{2}$, which means the required exposure reduced by over $80 \%$.

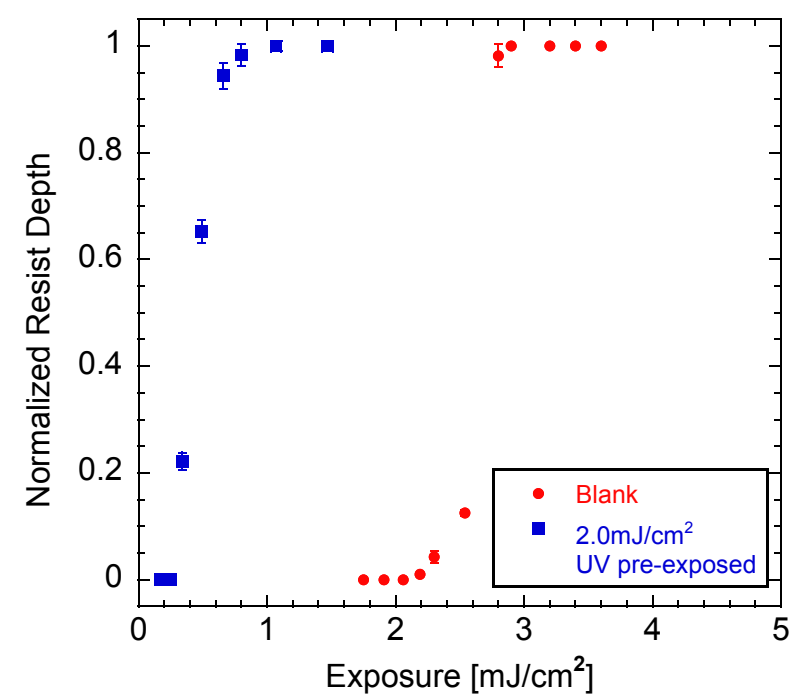

Figure 6. Sensitivity curve of $2.0 \mathrm{~mJ} / \mathrm{cm}^{2} \mathrm{UV}$ preexposed TDUR-P722 samples to deep-UV light

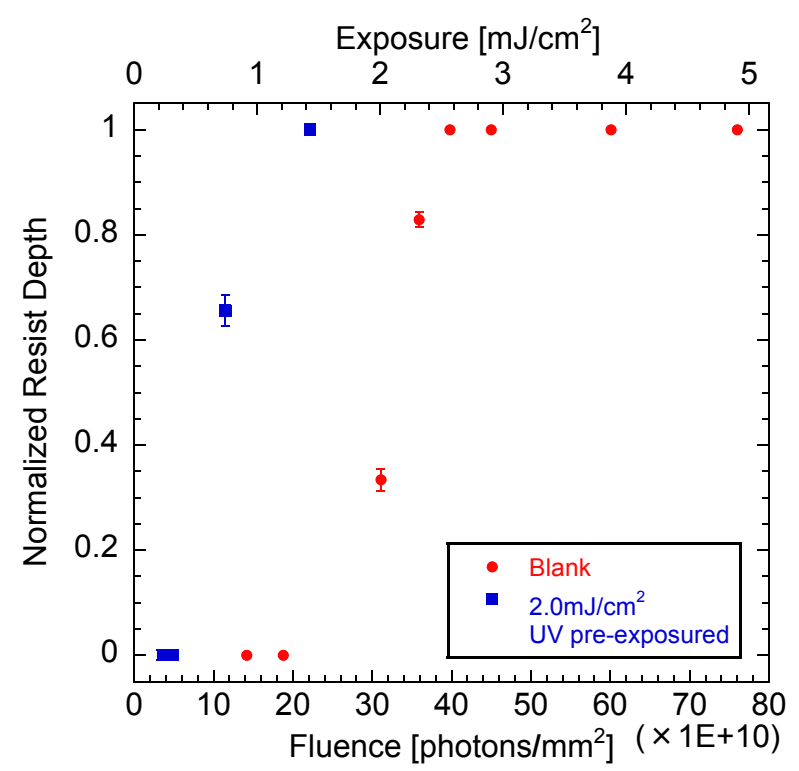

Figure 7. Sensitivity curve of $2.0 \mathrm{~mJ} / \mathrm{cm}^{2} \mathrm{UV}$ preexposed TDUR-P722 to $400 \mathrm{eV}$ soft X-ray exposure

This UV pre-exposure method was also confirmed very effective to the soft X-ray irradiation. After $2.0 \mathrm{~mJ} / \mathrm{cm}^{2}$ pre-exposure of the deep-UV light, the required irradiation amount of $400 \mathrm{eV}$ and $250 \mathrm{eV} \mathrm{X}$-rays reduced to about $1.0 \mathrm{E}+11$ photons $/ \mathrm{mm}^{2}\left(0.7 \mathrm{~mJ} / \mathrm{cm}^{2}\right)$ and $1.4 \mathrm{E}+12$ photons $/ \mathrm{mm}^{2}\left(5.6 \mathrm{~mJ} / \mathrm{cm}^{2}\right)$, respectively. The required $400 \mathrm{eV} \mathrm{X}$-ray amount successfully reduced by over $65 \%$. These results are adequate for contact microscopy with $100 \mathrm{~nm}$ resolution which is aimed at present, although the X-ray yield must be enhanced more for the practical use. 


\section{Conclusion}

At Waseda University, we have been developing a short-pulse inverse Compton soft X-ray source in the water window region. For the application to the soft X-ray microscopy, we are developing high resolution imaging system with photo resists. Because the generated X-ray yield had been too small for the practical use of the soft $\mathrm{X}$-ray microscopy, we have upgraded the generation system. The generated X-rays have successfully increased and the total generated $\mathrm{X}$-rays were estimated to be over $1.5 \mathrm{E}+5$ photon/s. Also, to reduce the required amount of X-rays, the super-sensitization of photo resists has been attempted. The photo sensitivity of a chemically amplified deep-UV photo resist, TDUR-P722, was evaluated in this study. We found that the UV pre-exposure method is one of the powerful tools for the super-sensitization, and it reduced the required $400 \mathrm{eV} \mathrm{X}$-ray amount by over $65 \%$.

\section{Acknowledgments}

The authors would like to express sincere thanks to Dr. Seiichi Tagawa and Dr. Kazumasa Okamoto of Osaka University for the fruitful discussion, and to the SAGA-LS staff members for their technical support.

This work has been supported by High-Tech Research Center Project of the Ministry of Education, Culture, Sports, Science and Technology (MEXT) HRC707 and JSPS Grant-in-Aid for Scientific Research (B)(2) 19340064. A part of this work was conducted at the BL12 in the SAGA Light Source (SAGA-LS), supported by Nanotechnology Network of MEXT, Japan.

\section{References}

1. S. Kashiwagi, Y. Hama, S. Hara, H. Ishikawa, R. Kuroda, T. Kobuki, T.
Oshima, K. Sato, M. Washio, A. Yada, J. Urakawa and H. Hayano, Int. J. Appl. Electromagnetics Mech., 14 (2001) 157 162.

2. S. Kashiwagi, R. Kuroda, T. Oshima, F. Nagasawa, T. Kobuki, D. Ueyama, Y. Hama, M. Washio, K. Ushida, H. Hayano and J. Urakawa, J. Appl. Phys., 98 (2005) 123302.

3. R.H. Milburn, Phys. Rev. Lett., 10 (1963) $75-77$

4. B. L. Henke, E. M. Gullikson and D. J. Davis, ADNDT, 54 (2) (1993) 181 - 342.

5. J. Kirz, C. Jacobsen and M. Howells, $Q$ Rev Biophys., 28 (1995) 33 - 130.

6. L Beese, R Feder, and D Sayre, Biophys J., 49 (1) (1986) 259 - 268.

7. Y. Yamamoto and K. Shinohara, Anat. Rec., 269 (5) (2002) 217 - 223.

8. D. Sayre, J. Kirz, R. Feder, D.M. Kim and E. Spiller, Ultramicroscopy, 2 (1977) 337-349

9. K. Shinohara and A. Ito, J Microsc., 161 (3) (1991) 463-472.

10. T. Gowa, A. Masuda, R. Moriyama, K. Sakaue, Y. Kamiya, M. Washio, R. Kuroda, S. Kashiwagi, K. Ushida, H. Hayano and J. Urakawa, Proc. PAC '07, (2007) 1031 1033.

11. K. Sakaue, T. Gowa, H. Hitoshi, K. Yoshio, S. Kashiwagi, R. Kuroda, A. Masuda, R. Moriyama, J. Urakawa, K. Ushida, X. J. Wang and M. Washio, Rad. Phy. Chem., 77 (2008) 1136 - 1141

12. The Kyushu Synchrotron Light Research Center, "SAGA Light Source", http://www.saga-ls.jp/

13. A. Masuda, T. Gowa, C. Igarashi, T. Kashino, N. Mitsuda, K. Sakaue, M. Washio, R. Kuroda, S. Kashiwagi, H. Hayano, J. Urakawa and K. Ushida, Proc. EPAC '08, (2008) 166-168. 